Meta

Journal des traducteurs

Translators' Journal

\title{
La synonymie en intelligence artificielle
}

\section{Ieda Maria Alves}

Volume 39, numéro 4, décembre 1994

Hommage à Bernard Quemada : termes et textes

URI : https://id.erudit.org/iderudit/001882ar

DOI : https://doi.org/10.7202/001882ar

Aller au sommaire du numéro

Éditeur(s)

Les Presses de l'Université de Montréal

ISSN

0026-0452 (imprimé)

1492-1421 (numérique)

Découvrir la revue

Citer cet article

Alves, I. (1994). La synonymie en intelligence artificielle. Meta, 39(4), 643-650. https://doi.org/10.7202/001882ar

\section{Résumé de l'article}

Dans cet article, nous exposons quelques problèmes concernant l'usage de synonymes en langue de spécialité. Les exemples présentés sont extraits d'un corpus portugais-brésilien de termes de l'intelligence artificielle. Quelques théories terminologiques n'acceptent pas l'occurrence de " termes synonymes », à cause de l'association univoque établie entre une notion et son terme correspondant ; d'autres théories, au contraire, reconnaissent que, d'après les contextes, une notion peut revêtir des formes synonymiques. Dans le corpus de l'intelligence artificielle que nous sommes en train d'analyser, environ $15 \%$ des termes dépouillés sont susceptibles d'avoir des synonymes ou des quasi-synonymes. Ce rapport synonymique peut être géré par différentes formes d'alternance : formation syntagmatique / terme formellement simple ; terme emprunté / terme indigène ; terme simple / synonyme contextuel ; formation syntagmatique / siglaison. Ces différentes possibilités d'association entre notions et termes démontrent que la synonymie en langue de spécialité existe et mérite d'être étudiée de plus près.
Ce document est protégé par la loi sur le droit d'auteur. L’utilisation des services d’Érudit (y compris la reproduction) est assujettie à sa politique d'utilisation que vous pouvez consulter en ligne.

https://apropos.erudit.org/fr/usagers/politique-dutilisation/ 


\title{
LA SYNONYMIE EN INTELLIGENCE ARTIFICIELLE
}

IEDA MARIA ALVES

Université de São Paulo, São Paulo, Brésil

\begin{abstract}
Résumé
Dans cet article, nous exposons quelques problèmes concernant l'usage de synonymes en langue de spécialité. Les exemples présentés sont extraits d'un corpus portugais-brésilien de termes de l'intelligence artificielle.

Quelques théories terminologiques n'acceptent pas l'occurrence de «termes synonymes», à cause de l'association univoque établie entre une notion et son terme correspondant ; d' autres théories, au contraire, reconnaissent que, d'après les contextes, une notion peut revêtir des formes synonymiques.

Dans le corpus de l'intelligence artificielle que nous sommes en train d'analyser. environ $15 \%$ des termes dépouillés sont susceptibles d'avoir des synonymes ou des quasisynonymes. Ce rapport synonymique peut être géré par différentes formes d'alternance : formation syntagmatique / terme formellement simple ; terme emprunté / terme indigène ; terme simple / synonyme contextuel; formation syntagmatique / siglaison.

Ces différentes possibilités d'association entre notions et termes démontrent que la synonymie en langue de spécialité existe et mérite d'être étudiée de plus près.
\end{abstract}

\begin{abstract}
In this article, we examine some problems related to the usage of synonyms in languages for special purposes (LSP). The examples presented are taken from a corpus of Brazilian Portuguese terms on Artificial Intelligence.

Some terminological theories do not accept the occurrence of "synonymic terms", based on the notion of univocal association between one concept and one term, while other theories recognize that concepts may occur in different contexts and that they may present different forms.

In the analysed LSP, approximately $15 \%$ of the collected terms are susceptible of having synonyms or quasi-synonyms. This synonymic relationship may be generated by different kinds of alternances: a syntagmatic unity/one form or a reduced syntagm; a borrowed term / a native term; a term / a contextual synonym; a syntagmatic unity / an abbreviated form.

These different possibilities of association between concepts and terms provide convincing evidence that synonyms do exist in LSP and merit a more thorough and detailed investigation.
\end{abstract}

\section{INTRODUCTION}

Ce travail prend sa source dans un accord établi, depuis 1990, entre l'Université de São Paulo (Brésil) et le Centre de Terminologie et de Néologie de l'Institut de la Langue Française (France), en vue de la publication d'un dictionnaire terminologique sur l'intelligence artificielle (IA), qui comprendra aussi des équivalents du portugais brésilien.

En ce qui concerne le corpus brésilien, les termes de l'IA sont en train d'être extraits de livres, actes de congrès, thèses et articles de revues consacrés à l'informatique et, spécialement, à l'intelligence artificielle.

Dans cet article, nous exposons les problèmes que nous rencontrons à propos de termes de l'intelligence artificielle qui, bien qu'ayant des signifiants différents, présentent les mêmes caractères et sont définis de manière identique. Sont-ils toujours des synonymes? 
Nous présentons, dans un premier temps, quelques considérations concernant les différentes opinions à l'égard de la synonymie en terminologie et, dans un second temps, nous analysons la problématique de la synonymie - ou non-synonymie - dans le corpus de l'intelligence artificielle que nous sommes en train de dépouiller.

En langue de spécialité, la synonymie est généralement traitée comme une question qui prête à confusion et qu'il faut essayer d'éviter. À ce propos, Felber (1987: 150) affirme que «les synonymes sèment la confusion et donnent l'impression fausse qu'il existe plus d'une notion» et il nous rappelle que «la communication technique est affaire de clarté plutôt que de variété». Chez Wüster, également, la valeur de la synonymie est niée, de même que l'homonynie et la polysémie: «ll ne devrait donc y avoir ni dénomination plurivalente (homonymes et polysèmes) ni dénominations multiples d'une même notion (synonymes)» (Einführung in die allgemeine Terminologielehre und terminologische Lexikographie, 1979, cité par Kocourek 1982a: 162).

Cette conception de la synonymie en langue de spécialité est la conséquence, on le sait, de l'idéal de l'univocité, c'est-à-dire le rapport unique établi entre signifiant et signifié ou entre dénomination et notion. D'où — on l'a maintes fois souligné (Dubuc 1982: 193 ; Rondeau 1984: 123, entre autres) - une distinction entre langue générale et langue de spécialité, car en langue générale, la synonymie est même considérée comme douée d'une valeur stylistique.

Malgré l'idéal d'univocité entre dénomination et notion, la synonymie en langue de spécialité est reconnue par la plupart des banques de termes: dans onze grandes banques de données étudiées par Felber (Normaterm, France; Din/Term, RFA; BTQ, Canada; Termium II, Canada; Eurodicautom, Communautés Européennes; ASITO, Russie; Team, RFA; Lexis, RFA; EWF, RDA; Termdok, Suède et ONU), le champ synonymie n'est absent que d'une banque (l'ASITO), dont les terminologues n'acceptent pas le rapport de synonymie en terminologie (op. cit.: 251 ; voir aussi Lerat 1988: 17).

Défini dans la norme ISO 1087, le terme synonymie y apparaît comme la «relation entre désignations de même langue qui représentent la même notion». Une note donne des renseignements supplémentaires et établit une différence entre synonymes et quasisynonymes: «Les termes qui sont interchangeables dans tous les contextes d'un domaine se nomment synonymes; s'ils ne sont interchangeables que dans certains contextes, ils sont appelés quasi-synonymes». Dans le Vocabulaire systématique de la terminologie (1985: 21), les auteurs sont très explicites à propos des différences qui distinguent un synonyme d'un quasi-synonyme : les termes synonymes désignent une même notion et se situent à un même niveau de langue ou à un même niveau de conceptualisation, tandis que les quasi-synonymes, qui désignent aussi une même notion, se situent à des niveaux de langue ou à des niveaux de conceptualisation différents ou s'emploient dans des situations de communication différentes. En guise de note, sont mentionnés les différents types de quasi-synonymes: quasi-synonymes de niveau (mal de tête en langue générale et céphalée en langage médical); quasi-synonymes géographiques (scie à chaîne au Canada et tronçonneuse en France); quasi-synonymes temporels (aéroplane et avion); quasi-synonymes professionnels (acide chlorhydrique en chimie et acide muriatique en maçonnerie); quasisynonymes de concurrence (pompe à chaleur et pompe thermique); quasi-synonymes de fréquence (rhume de cerveau et coryza). Ces types de quasi-synonymes correspondent à la classification en synonymes proposée par Dubuc (1978: 59-63).

Felber considère aussi ce rapport entre notion et terme comme relevant de la synonymie (op. cit. : 154). Il énumère les causes de l'utilisation de synonymes en terminologie en nous montrant que cette synonymie est toujours causée par l'utilisation parallèle de deux termes: un terme indigène et un terme international (matter/substance, en anglais), un terme indigène et un terme emprunté (writing desk/bureau, en anglais), un terme 
général et un terme scientifique (mal de tête/céphalée) ou par l'utilisation parallèle du nom d'un inventeur et d'un caractère intrinsèque ou extrinsèque du terme (effet Johnson / effet thermique), d'un terme et d'un symbole ou d'une abréviation (eau/H2O) ou d'un nom de marque et d'un terme scientifique (aspirine/acide acétylsalicylique). L'étude des causes de la synonymie en langue de spécialité a occupé d'autres auteurs, comme Corbeil (1974: 60-65). Ce terminologue prend en considération quelques raisons déjà exposées par Felber (utilisation parallèle d'un terme indigène et d'un emprunt, existence de niveaux de langue) et en outre il énumère d'autres causes qui provoquent l'emploi de termes synonymiques: concurrence morphologique entre des expressions syntagmatiques et des formes simples; concurrence des théories, des techniques, des procédés, des établissements ou des entreprises; nouveauté du référent; imprécision de la notion. Sager (1990: 214215) traite également du sujet, en ajoutant que la concurrence morphologique entre un terme ou expression syntagmatique et sa forme abrégée, peut être causée par l'omission d'un élément du syntagme ou par la réduction du terme. Les causes de l'emploi synonymique en terminologie peuvent aussi être dues à une création alternative, motivée par l'ignorance du terme adéquat de la part de traducteurs et de professionnels d'un domaine et même à une normalisation sans succès (Picht et Draskau 1985: 103).

\section{LE CHAMP DE LA SYNONYMIE EN INTELLIGENCE ARTIFICIELLE}

La relation de synonymie entre deux termes ne peut être établie qu'après l'élaboration de leurs définitions, car deux termes ne sont synonymes que s'ils désignent la même notion, ou, en d'autres termes, si leurs notions révèlent une même compréhension et une même extension.

Dans le corpus de la terminologie de l'intelligence artificielle que nous analysons (à peu près 1000 termes), la définition de quelque 450 termes nous démontre qu'environ $15 \%$ de ces termes définis établissent des rapports qu'on peut, dans un premier temps, considérer comme synonymiques ou quasi synonymiques.

Ces rapports sont dus, d'après notre observation, à la caractérisation formelle des unités lexicales en langue de spécialité, laquelle privilégie la formation de nouvelles unités, ou des néonymes, selon certains modes de formation, spécialement la dénomination syntagmatique ${ }^{1}$.

Les formations syntagmatiques, les termes-syntagmes, constituent, dans toutes les terminologies, un procédé de formation très productif. Ce fait est à l'origine de synonymies, étant donné qu'un syntagme formé d'un terme générique et d'une expansion peut être concurrencé par d'autres syntagmes ou par une forme monolithique.

Les exemples de l'intelligence artificielle dont nous disposons sont abondants et revêtent plusieurs formes.

Des syntagmes commençant par des termes génériques tels que méthode, problème, procédé, procès, système..., tendent à présenter des synonymes, syntagmatiques également, dépourvus de ces noyaux génériques. Le noyau générique de la forme alternante devient, par conséquent, l'élément qui était le centre du syntagme prépositionnel:

- mecanismo de pesquisa heurística/pesquisa heurística;

- problema de satisfação de restriçôes/satisfação de restrições ;

- procedimento de busca minimax / busca minimax.

La synonymie établie entre deux syntagmes, chacun d'entre eux pourvus de différents termes génériques, peut provenir d'une association synonymique existante en langue générale:

resolvedor geral de problemas/solucionador geral de problemas ; 
ou, fréquemment, d'une alternance de caractère hyperonymique (máquina, método) versus hyponymique (computador, busca):

- máquina inteligente/computador inteligente;

- método em profundidade / busca em profundidade ;

ou même d'une confusion terminologique caractéristique du sous-domaine:

- banco de dados relacional / base de dados relacional.

Le rapport synonymique résulte aussi d'une simplification du syntagme. Le noyau générique disparaît et son expansion, le déterminant spécifique, de caractère adjectival, s'oppose à une unité terminologique sous forme substantivale :

- fórmula atômica / átomo;

- processo dedutivo/dedução.

D'autres possibilités de réduction syntagmatique raccourcissent le syntagme, en le réduisant soit au terme générique qui, lui seul, passe à désigner la notion correspondante :

- interpretador de regras / interpretador

- lógica simbólica / lógica;

- perceptron de duas camadas / perceptron;

soit à un terme formellement différent :

- átomo simbólico/constante.

Dans quelques formations syntagmatiques on remarque que la synonymie n'existe qu'au niveau des caractères déterminants, qu'ils soient des adjectifs synonymes:

- grafo dirigido/grafo orientado;

- lógica proposicional/lógica sentencial;

- prova mecânica de teorema / prova automática de teorema;

- teoria dos conjuntos nebulosos/teoria dos conjuntos_difusos;

des noms synonymes :

- árvore de busca lárvore de pesquisa, árvore de procura ;

- reconhecimento da fala / reconhecimento da voz;

- regra de dedução/regra de inferência;

- sistema de apoio à decisáo / sistema de suporte à decisão ;

ou des mots de la même famille étymologique:

- explosão combinatória / explosão combinatorial ;

- forma clausular / forma de cláusula ;

- rede neural/ rede neuronal.

L'opposition entre les déterminants peut aussi refléter l'emploi d'un adjectif de la langue générale vis-à-vis d'un syntagme prépositionnel où l'élément nominal désigne un objet matériel:

- tradução automática / tradução por máquina;

- visão artificial/ visão por computador, visão de máquina.

En intelligence artificielle, des formations syntagmatiques sont souvent employées sous forme de sigle (voir, pour le français, Pavel 1988:1-4). La dénomination de la 
science elle-même (IA), ses programmes les plus répandus (LISP, Prolog), ainsi que quelques-uns de ses termes, connaissent un concurrent sous la forme de la siglaison:

- fator de certeza/FC;

- Inteligência Artificial/IA ;

- List Processing Language/LISP ;

- Programming in Logic/Prolog ;

- rede semântica intensional /RSI;

- sistema especialista/SE, SES, SEs;

- sistema especialista baseado em conhecimento/SBC;

ou partiellement sous cette forme:

- arquitetura do sistema especialista / arquitetura do SE.

Outre les formations syntagmatiques, l'opposition synonymique en intelligence artificielle caractérise aussi des termes formellement simples, des dérivés et des composés².

Le même radical peut dériver des termes qui ne se différencient que par le formant, en donnant lieu à ce que Kocourek (op. cit.:164) définit comme la synonymie des formants. - ção et -mento, des suffixes concurrents qui, à partir de bases verbales, forment des noms qui désignent une action ou son résultat, dérivent le couple concurrent monitoração/monitoramento.

Des termes morphologiquement composés tels que espaço-problema, nó-filho, nó-pai et nó-raiz s'opposent à une formation syntagmatique (espaço de problema) ou à des formes simples (filho, pai, raiz) qui se débarrassent de l'élément déterminé et dont l'élément déterminant, métaphorique, passe à désigner, lui seul, la notion ${ }^{3}$.

Des termes morphologiquement simples s'opposent synonymiquement à d'autres formes simples dans des cas d'emprunts, très fréquents en intelligence artificielle, soit à la langue générale:

- fato/dado;

- nó/nódulo

soit à d'autres langues de spécialité :

- antecedente/premissa (philosophie) ;

- arborescência /árvore (morphologie végétale);

- axioma/postulado (philosophie).

L'association synonymique se révèle également entre une unité lexicale de la langue générale et un terme d'une langue de spécialité:

- conclusão (langue générale)/consequente (philosophie).

Une autre cause de l'emploi de synonymes dans les langues de spécialité est constituée par l'utilisation parallèle d'un terme étranger, d'habitude anglais, et d'un terme autochtone.

En portugais brésilien, la terminologie de l'informatique est une source constante de néonymes empruntés à l'anglais, qui provoquent, en plus, de curieuses formations hybrides (delet - portugais deletar, par exemple). Dans le sous-domaine de l'intelligence artificielle, au contraire, les termes anglais sont assez rares et sont toujours concurrencés par des équivalents portugais, formés en accord avec les règles morphologiques de la langue portugaise, ces derniers se faisant eux-mêmes concurrence :

- backtracking/rastreamento para trás;

- backward chaining / encadeamento para trás, encadeamento regressivo, encadeamento retroativo; 
backward reasoning / inferência para trás, processo dirigido por objetivos ;

blackboard / quadro-negro;

- breadth-first / busca em nivel, busca em amplitude;

- certainty factor / fator de certeza, fator de confiança;

- depth-search, depth-first search / busca em profundidade ;

- fork/nó-forquilha;

- forward chaining / encadeamento para frente, encadeamento progressivo;

- forward reasoning / inferência para frente, raciocínio para frente;

- frame lestante, quadro;

- generate and test/gerar e testar;

- hill-climbing / busca subindo morro, subida de encosta;

- matching / casamento;

- script/roteiro,

- state space lespaço de estados;

- well-formed formula / fórmula bem-formada.

Le corpus étudié présente aussi trois termes, de caractère syntagmatique, ayant le déterminé générique en portugais et le déterminant en anglais :

- ârvore and/or;

- grafo and/or;

informação fuzzy;

et, de manière analogue aux emprunts cités ci-dessus, ils subissent la concurrence des équivalents portugais :

- árvore elou;

- grafo elou;

dado incerto.

À part les emprunts proprement dits, ou xénotermes d'après Boulanger (1987: 328329), la terminologie de l'intelligence artificielle présente des calques: regra se...então (anglais rule if...then, français règle si...alors), par exemple. Très difficile à repérer, le terme calqué est un emprunt sans être l'emprunt. C'est pour les traducteurs et les terminologues un vrai miroir aux alouettes lexicales (idem, ibidem).

Dans le travail mentionné, Boulanger cite des termes français de l'intelligence artificielle, calqués en anglais, qui respectent la structure française déterminé-déterminant. La même remarque peut être appliquée au portugais, et des termes tels que encadeamento para trás (anglais backward chaining, français chaînage arrière), explosão combinatória (angl.combinatorial explosion, fr. explosion combinatoire), motor de inferência (ang1. inference engine, fr. moteur d'inférence), engenheiro do conhecimento (angl. knowledge engineer, fr. ingénieur de la connaissance), sistema especialista (angl. expert system, fr. système expert) ne sont plus sentis comme provenant d'une source non autochtone.

\section{NOTES FINALES}

L'analyse que nous avons effectuée nous a persuadée que, en langue de spécialité, les rapports synonymiques existent et sont justifiables, dans certains cas.

«Le terme scientifique et technique n'admet pas de synonymie autre que référentielle», nous dit-on (Guilbert 1973: 11). La synonymie en langue de spécialité, libre de connotations, porte donc, spécifiquement, sur la dénomination. Et de cette perspective, la concurrence entre un terme indigène et un terme étranger, entre un terme standard et son emploi argotique, entre une expression syntagmatique et une forme simple ne nous paraît pas un signe de confusion, mais plutôt une conséquence du fait que la notion a un caractère universel et n'est pas liée aux langues individuelles. 
L'activité normalisatrice, souvent citée comme une opération apte à éliminer les rapports synonymiques (Duchacek op. cit.: 118; Dubuc 1982: 201; Rey 1982:301) n'est pas toujours assez puissante pour réduire la relation notion/terme. À ce propos, Rey (idem, ibidem) nous rappelle que la normalisation doit aussi tenir compte de la nature des systèmes notionnels concernés, de leur production et de leur fonctionnement, très divers et parfois opposés. Depecker (1990: 204-206) considère que la synonymie peut être très précieuse, car le terme synonyme conserve la trace d'une démarche conceptuelle ou de son origine - étrangère, géographique ou morphologique.

En terminologie bilingue, l'existence des rapports synonymiques est plutôt utile. Lerat (op. cit.: 23) nous signale que, dans la terminologie orientée vers la traduction, l'existence des synonymes se révèle un moyen rapide de contrôle du sens dans les deux langues.

Il est certain que, parmi les termes de l'intelligence artificielle que nous avons considérés comme ayant des rapports synonymiques, et dont nous avons donné quelques exemples, tous ne pourront pas, à la suite d'une analyse plus fine, être retenus comme des synonymes. Il faut y déceler des variantes morphologiques, plutôt que synonymiques (c'est le cas de monitoramento/monitoração, par exemple), des termes appartenant à différents niveaux linguistiques, des termes d'usage plus fréquent, des termes qui ne sont interchangeables que dans certains contextes - des quasi-synonymes - et, pour ces éléments, notre fiche terminologique doit abriter un nouveau champ: celui de la quasisynonymie.

En guise de conclusion, et en justifiant l'emploi de synonymes en langue de spécialité, reprenons Kocourek (1982b: 257-258) qui nous dit que «l'idéal de l'absence de synonymie n'est pas le seul principe de la langue techno-scientifique. Il y a, également, l'idéal de la sauvegarde de la multiplicité et de la flexibilité des fonctions et de la liberté de l'expérimentation individuelle. Ces idéals permettent d'assurer une connaissance technoscientifique un peu plus nuancée, plus complète, plus objective et plus applicable».

\section{Notes}

1. Otto Duchacek présente, dans «La synonymie en terminologie», trois types selon lesquels les termes synonymiques peuvent être répartis: 1 . avec le membre déterminé commun; 2 . avec le membre déterminant commun ; 3. sans membre commun (1977:112-116).

2. Il s'agit, dans tous ces cas, de ce que Lethuillier (1989: 443-449) définit comme la synonymie de forme.

3. Kocourek (1982a : 165) énumère des exemples, pris au français, de ce type d'opposition formelle : la formation syntagmatique (science du sol) / la confixation (pédologie); la formation syntagmatique (secrétaire de tournage)/l'emprunt (script-girl); un affixe (dyoxide, modificateur)/un autre affixe (bioxyde, modifieur)...

\section{RÉFÉRENCES}

BOULANGER, Jean-Claude (1987) : «Le miroir aux alouettes en intelligence artificielle», Meta, 32-3, Montréal, Presses de l'Université de Montréal, pp. 326-331.

BOUTIN-QUESNEL, Rachel et al. (1985) : Vocabulaire systématique de la terminologie, Québec, Office de la langue française.

CORBEIL, Jean-Claude (1974) : «Problématique de la synonymie en vocabulaire spécialisé», La Banque des mots, 7, pp. 53-58.

DEPECKER, Loïc (1990) : «Éloge de la synonymie», Actas do Colóquio de Lexicologia e Lexicografia, Lisboa, pp. 204-206.

DUBUC, Robert (1978): Manuel pratique de terminologie, Québec, Linguatech/Conseil international de la langue française.

DUBUC, Robert (1982) : «Synonymie et terminologie», Problèmes de la définition et de la synonymie en terminologie. Actes du Colloque international de terminologie, Québec, pp. 193-206.

DUCHACEK, Otto (1977): «La synonymie en terminologie», Actes du $6^{\circ}$ Colloque international de terminologie, Pointe-au-Pic, Québec, pp. 107-118.

FELBER, Helmut (1987): Manuel de terminologie, Paris, Unesco/Infoterm. 
GUILBERT, Louis (1973) : «La spécificité du terme scientifique et technique», Langue française, 17-2, pp. 5-17. KOCOUREK, Rostislav (1982a): La langue française de la technique et de la science, Paris/Wiesbaden, La Documentation Française / Oscar Brandstetter.

KOCOUREK, Rostislav (1982b) : «Rapports entre la synonymie en terminologie et la délimitation des notions», Problèmes de la définition et de la synonymie en terminologie. Actes du Colloque international de terminologie, Québec, pp. 20-65.

LERAT, Pierre (1988): «Terminologie et sémantique descriptive», La Banque des mots, numéro spécial, pp. 11-30.

LETHUILLIER, Jacques (1989): «La synonymie en langue de spécialité», Meta, 34-4, Montréal, Presses de l'Université de Montréal, pp. 443-449.

Norme internationale ISO 1087 (1990).

PAVEL, Silvia (1988): «Siglaison et créativité lexicale en intelligence artificielle», L'actualité terminologique, 21 , pp. $1-4$.

PICHT, Herbert et Jennifer DRASKAU (1985) : Terminology : an Introduction, Surrey, University of Surrey.

REY, Alain (1982): «Synonymie, néonymie et normalisation terminologique», Problèmes de la définition et de la synonymie en terminologie. Actes du Colloque international de terminologie, Québec, pp. 282-310.

RONDEAU, Guy (1984) : Introduction à la terminologie, Québec, Gaëtan Morin.

SAGER, Juan C. (1990) : A Practical Course in Terminology Processing, Amsterdam/Philadelphia, John Benjamins. 